\title{
LINE ELEMENT FIELDS ON MANIFOLDS
}

BY

\author{
W. S. MASSEY AND R. H. SZCZARBA(1)
}

Introduction. The purpose of this note is to investigate the characteristic classes of manifolds admitting line element fields. Our main result, stated in $\$ 1$, asserts that, if a compact $n$-dimensional manifold has $k$ independent line element fields, then the characteristic classes can be expressed in terms of lower dimensional elements. In particular, if $k=n$, the Stiefel-Whitney classes are expressible as elementary symmetric functions of one dimensional classes and the Pontrjagin classes as elementary symmetric functions of the Bocksteins of these one dimensional classes. In $\S 3$, we give necessary and sufficient conditions that an orientable four dimensional manifold have four independent line element fields. As an application, we give an example of a fourdimensional non-parallelizable orientable manifold with four independent line element fields.

1. The main theorems. Let $O(n)$ denote the orthogonal group of $n \times n$ matrices, $S O(n) \subset O(n)$ the special orthogonal group, and $Q(n), S Q(n)$ the subgroups of diagonal matrices in $O(n), S O(n)$ respectively. Clearly $Q(n)$ $\approx Z_{2}^{n}$ and $S Q(n) \approx Z_{2}^{n-1}$.

For each integer $k, 0<k \leqq n$, we define a subgroup $G(k) \subset O(n)$ to consist of all matrices of the form

$$
\left(\begin{array}{lllll}
A & & & & 0 \\
& & & & \\
0 & \epsilon_{1} & & & 0 \\
& & & \cdot & \\
& & 0 & \cdot & \\
& & & & \epsilon_{k}
\end{array}\right)
$$

where $A \in O(n-k)$ and $\epsilon_{i}= \pm 1,1 \leqq i \leqq k$. Let $S G(k)=G(k) \cap S O(n)$ and $F_{k}=\boldsymbol{O}(n) / \boldsymbol{G}(k)=S O(n) / S G(k)$. Clearly $\boldsymbol{G}(k) \approx O(n-k) \times Z_{2}^{k}, S G(k)$ $\approx O(n-k) \times Z_{2}^{k-1}$, and both $G(k)$ and $S G(k)$ act on $F_{k}$.

Some other notation we will use is as follows. For any fiber bundle $\zeta=(E, \pi, B)$ with fiber $F$ and group $O(n), w_{j}(\zeta) \in H^{j}\left(B ; Z_{2}\right), 1 \leqq j \leqq n$, will denote the $j$ th Stiefel-Whitney class of $\zeta, p_{j}(\zeta) \in H^{4 j}(B ; Z), 1 \leqq j \leqq n / 2$, the $j$ th Pontrjagin class of $\zeta$, and, if $\zeta$ is orientable, $\chi(\zeta) \in H^{n}(B ; \boldsymbol{Z})$ the EulerPoincaré class of $\zeta$. For any compact $n$-dimensional manifold $M, w_{j}(M)$,

Received by the editors October 9, 1961.

(1) During the preparation of this paper, the second named author was partially supported by O.N.R. contract SAR/NOR(G) 00012-60. 
$1 \leqq j \leqq n, p_{j}(M), 1 \leqq j \leqq n / 4$, and, if $M$ is orientable, $\chi(M)$ will denote the corresponding classes of the tangent bundle of $M$. Further, $\sigma_{r}\left(x_{1}, \cdots, x_{n}\right)$ will denote the $r$ th elementary symmetric function of $x_{1}, \cdots, x_{n}$ with $\sigma_{0}\left(x_{1}, \cdots, x_{n}\right)=1, \sigma_{r}\left(x_{1}, \cdots, x_{n}\right)=0$ if $r<0$ or $r>n$. Finally, for any space $X, \delta^{*}: H^{q}\left(X ; \boldsymbol{Z}_{2}\right) \rightarrow H^{q+1}(X ; \boldsymbol{Z})$ will denote the Bockstein homomorphism.

TheOREm A. Let $\zeta=(E, \pi, B)$ be a fiber bundle with fiber $F_{k}$ and group $O(n)$. Then, if $\zeta$ has a cross section, there exist elements $x_{1}, \cdots, x_{k} \in H^{1}\left(B ; Z_{2}\right)$, $u_{j} \in H^{j}\left(B ; \boldsymbol{Z}_{2}\right), 1 \leqq j \leqq n-k$, and $v_{j} \in H^{4 j}(B ; \boldsymbol{Z}), 1 \leqq j \leqq n-k / 2$, such that

$$
\begin{aligned}
w_{q}(\zeta)= & \sum_{i+j=q} u_{i} \sigma_{j}\left(x_{1}, \cdots, x_{k}\right), \quad 1 \leqq q \leqq n \\
p_{q}(\zeta)= & \sum_{i+j=q}\left\{v_{i} \sigma_{2 j}\left(\delta^{*} x_{1}, \cdots, \delta^{*} x_{k}\right)\right. \\
& \left.\quad+\left[\left(\delta^{*} u_{2 i}\right)^{2}+\delta^{*} u_{1} v_{i}\right] \sigma_{2 j-1}\left(\delta^{*} x_{1}, \cdots, \delta^{*} x_{k}\right)\right\}, \quad 1 \leqq q \leqq n / 2 .
\end{aligned}
$$

Furthermore, if $\zeta$ is orientable $\left({ }^{2}\right)$,

$$
n \chi(\zeta)=\delta^{*} w_{n-1}(\zeta)
$$

Let $M$ be a compact $n$-dimensional manifold with tangent bundle $\tau(M)$. It is obvious that $M$ has $k$ independent line element fields if and only if the bundle with fiber $F_{k}$ associated with $\tau(M)$ has a cross section. Thus we have

COROLlARY 1. Let $M$ be a compact $n$-dimensional manifold. Then, if $M$ has $k$ independent line element fields, there exist elements $x_{1}, \cdots, x_{k} \in H^{1}\left(M ; Z_{2}\right)$, $u_{j} \in H^{j}\left(M ; Z_{2}\right), 1 \leqq j \leqq n-k$, and $v_{j} \in H^{4 j}(M ; Z), 1 \leqq j \leqq n-k / 4$, such that

$$
\begin{aligned}
w_{q}(M)= & \sum_{i+j=q} u_{i} \sigma_{j}\left(x_{1}, \cdots, x_{k}\right), \quad 1 \leqq q \leqq n, \\
p_{q}(M)= & \sum_{i+j=q}\left\{v_{i} \sigma_{2 j}\left(\delta^{*} x_{1}, \cdots, \delta^{*} x_{k}\right)\right. \\
& \left.\quad+\left[\left(\delta^{*} u_{2 i}\right)^{2}+\delta^{*} u_{1} v_{i}\right] \sigma_{2 j-1}\left(\delta^{*} x_{1}, \cdots, \delta^{*} x_{k}\right)\right\}, \quad 1 \leqq q \leqq n / 4 .
\end{aligned}
$$

Furthermore, if $M$ is orientable $\left({ }^{3}\right), \chi(M)=0$.

The first two statements of Corollary 1 are immediate from Theorem A. The last follows from the fact that $2 n \chi(M)=0$ but $H^{n}(M ; Z)$ is torsion free.

In particular, if $M$ has $n$ independent line element fields, we say that $M$ is line element parallelizable and we have

Corollary 2. If $M$ is line element parallelizable, there are elements $x_{1}, \cdots, x_{n} \in H^{1}\left(M ; Z_{2}\right)$ such that

$$
\begin{aligned}
& w_{q}(M)=\sigma_{q}\left(x_{1}, \cdots, x_{n}\right), \quad 1 \leqq q \leqq n, \\
& p_{q}(M)=\sigma_{2 q}\left(\delta^{*} x_{1}, \cdots, \delta^{*} x_{n}\right), \quad 1 \leqq q \leqq n / 4 .
\end{aligned}
$$

(2) Note that, for $n$ odd, one always has $\chi(\zeta)=\delta^{*} w_{n-1}(\zeta)$ so (1.3) is redundant in this case.

${ }^{(3)}$ The fact that the Euler-Poincaré class of an orientable manifold admitting a line element field is zero follows from Samelson [6] or Marcus [4]. 
Furthermore, if $M$ is orientable, $\chi(M)=0$.

REMARK 1. We note that, although (1.6) does follow from Theorem A, it is in fact a trivial consequence of Borel's work [1].

REMARK 2. We give an interpretation to the $x_{1}, \cdots, x_{k}$ of Theorem $\mathrm{A}$, as follows. Let $\tilde{\zeta}=(\tilde{E}, \tilde{\pi}, B)$ be the bundle with fiber $O(n) / O(k)$ associated with $\zeta$. It is easily seen that we can choose $\tilde{E}$ to be a covering of $E$ with group $\boldsymbol{Z}_{\mathbf{2}}^{\boldsymbol{k}}$. Then, if $f: B \rightarrow E$ is a cross section in $\zeta,\left(x_{1}, \cdots, \boldsymbol{x}_{k}\right) \in H^{1}\left(B ; \boldsymbol{Z}_{2}\right) \oplus \cdots$ $\oplus H^{1}\left(B ; Z_{2}\right) \approx H^{1}\left(B ; Z_{2}^{k}\right)$ can be identified with the obstruction to lifting $f$ to a cross section $\tilde{f}: B \rightarrow \tilde{E}$ of $\tilde{\zeta}$.

2. Proof of Theorem A. Suppose $B_{O(n)}$ is the classifying space for $O(n)$ and $\phi: B \rightarrow B_{O(n)}$ the classifying map for the principal bundle associated with $\zeta$. Then $\zeta$ is induced by $\phi$ from the bundle $\alpha: B_{G(k)} \rightarrow B_{O(n)}$ ( $\alpha$ induced by the inclusion map $G(k) \subset O(n))$ and $\zeta$ has a cross section if and only if there is a $\operatorname{map} f: B \rightarrow B_{G(k)}$ such that $\alpha f \simeq \phi$.

Let $w_{i} \in H^{i}\left(B_{O(n)} ; \boldsymbol{Z}_{2}\right), 1 \leqq i \leqq n$, be the universal Stiefel-Whitney classes and $p_{i} \in H^{4 i}\left(B_{O(n)} ; Z\right), 1 \leqq i \leqq n / 2$, the universal Pontrjagin classes. Let $\tilde{w}_{i} \in H^{i}\left(B_{G(k)} ; Z_{2}\right), 1 \leqq i \leqq n-k$, and $\tilde{p}_{i} \in H^{4 i}\left(B_{G(k)} ; Z\right), 1 \leqq i \leqq n-k / 2$, be the images of the universal classes under the map $H^{*}\left(B_{O(n-k)}\right) \rightarrow H^{*}\left(B_{G(k)}\right)$ induced by the projection $G(k) \rightarrow O(n-k)$. Further, let $y_{1}, \cdots, y_{k} \in H^{1}\left(B_{G(k)} ; Z_{2}\right)$ be the images of the generators (described by Borel [1]) of $H^{1}\left(B_{Q_{(k)}} ; Z_{2}\right)$ under the map $H^{1}\left(B_{\boldsymbol{Q}_{(k)}} ; \boldsymbol{Z}_{2}\right) \rightarrow H^{1}\left(B_{\boldsymbol{G}(k)} ; \boldsymbol{Z}_{2}\right)$ induced by the projection $\boldsymbol{G}(k) \rightarrow \boldsymbol{Q}(k)$. Define $x_{i}=f^{*} y_{i}, 1 \leqq i \leqq k, u_{i}=f^{*} \tilde{w}_{i}, 1 \leqq i \leqq n-k$, and $v_{i}=f^{*} \tilde{p}_{i}, 1 \leqq i \leqq n-k / 2$. We now show that these elements satisfy the conditions of Theorem A. We first verify condition (1.1). Since $w_{q}(\zeta)=\phi^{*} w_{q}=f^{*} \alpha^{*} w_{q},(1.1)$ can be rewritten

$$
f^{*} \alpha^{*} w_{q}=f^{*} \sum_{i+j=q} \tilde{w}_{i} \sigma_{j}\left(y_{1}, \cdots, y_{n-k}\right)
$$

We in fact show that $\alpha^{*} w_{q}=\sum_{i+j=q} \tilde{w}_{i} \sigma_{j}\left(y_{1}, \cdots, y_{n-k}\right)$.

Consider the composite

$$
H^{q}\left(B_{O(n)} ; Z_{2}\right) \stackrel{\alpha^{*}}{\longrightarrow} H^{q}\left(B_{G(k)} ; Z_{2}\right) \stackrel{\beta^{*}}{\longrightarrow} H^{q}\left(B_{Q(n)} ; Z_{2}\right)
$$

where $\alpha$ and $\beta$ are induced by the inclusion maps $G_{k} \subset O(n)$ and $Q(n) \subset G(k)$. Borel [1] has shown that $\alpha^{*}$ and $\beta^{*}$ are 1-1 and that there are generators $z_{1}, \cdots, z_{n}$ of $H^{1}\left(B_{Q(n)} ; Z_{2}\right)$ such that $\beta^{*} \alpha^{*} w_{q}=\sigma_{q}\left(z_{1}, \cdots, z_{n}\right)$. Furthermore, it is easily seen that $z_{1}, \cdots, z_{n}$ can be chosen so that $\beta^{*} \tilde{w}_{i}=\sigma_{i}\left(z_{1}, \cdots, z_{n-k}\right)$, $1 \leqq i \leqq n-k$, and $\beta^{*} y_{j}=z_{n-k+j}, 1 \leqq j \leqq k$. Then

$$
\beta^{*} \sum_{i+j=q} \tilde{w}_{i} \sigma_{j}\left(y_{1}, \cdots, y_{k}\right)=\sum_{i+j=q} \sigma_{i}\left(z_{1}, \cdots, z_{n-k}\right) \sigma_{j}\left(z_{n-k+1}, \cdots, z_{n}\right)
$$

which, by a well known identity, is $\sigma_{q}\left(z_{1}, \cdots, z_{n}\right)$. Thus

$$
\beta^{*} \sum_{i+j=q} \tilde{w}_{i} \sigma_{j}\left(y_{1}, \cdots, y_{k}\right)=\beta^{*} \alpha^{*} w_{q}
$$


and since $\beta^{*}$ is $1-1$, the result is proved.

By a similar argument, (1.2) follows from the identity

$$
\begin{aligned}
\alpha^{*} p_{q}=\sum_{i+j=q}\left\{\tilde { p } _ { i } \sigma _ { 2 j } \left(\delta^{*} y_{1}, \cdots\right.\right. & \left., \delta^{*} y_{k}\right) \\
& \left.+\left[\left(\delta^{*} \tilde{w}_{2 i}\right)^{2}+\delta^{*} \tilde{w}_{1} \tilde{p}_{i}\right] \sigma_{2 j-1}\left(\delta^{*} y_{1}, \cdots, \delta^{*} y_{k}\right)\right\}
\end{aligned}
$$

which we now prove.

Consider the diagram

$$
\begin{gathered}
H^{q}\left(B_{G(k)} ; Z\right) \stackrel{\beta^{*}}{\longrightarrow} H^{q}\left(B_{Q_{(n)}} ; Z\right) \\
\quad r \downarrow \\
H^{q}\left(B_{G_{(k)}} ; Z_{2}\right) \stackrel{\beta^{*}}{\longrightarrow} H^{q}\left(B_{Q_{(n)}} ; Z_{2}\right)
\end{gathered}
$$

where $r$ is reduction mod 2. Since $2 H^{q}\left(B_{Q(n)} ; Z\right)=0$, it follows from the Bockstein exact sequence that $r: H^{q}\left(B_{Q(n)} ; \boldsymbol{Z}\right) \rightarrow H^{q}\left(B_{Q_{(n)}} ; Z_{2}\right)$ is $1-1$. We denote the right side of $(2.1)$ by $M_{q}$ and compute $r \beta^{*} M_{q}$. Note first of all that $r \beta^{*} \delta^{*} y_{j}=\beta^{*} r \delta^{*} y_{j}=\beta^{*} S_{q}^{1} y_{j}=\beta^{*} y_{j}^{2}, r \beta^{*} \tilde{w}_{j}=\sigma_{j}\left(z_{1}, \cdots, z_{n-k}\right)$, and $r \beta^{*} \tilde{p}_{j}=\beta^{*} r \tilde{p}_{j}$ $=\beta^{*} \tilde{w}_{2 j}^{2}=\sigma,{ }_{, j}\left(z_{1}, \cdots, z_{n-k}\right)^{2}$ so

$$
\begin{array}{r}
r \beta^{*} M_{q}=\sum_{i+j=q}\left\{\sigma_{2 i}\left(z_{1}, \cdots, z_{n-k}\right) \sigma_{2 j}\left(z_{n-k+1}, \cdots, z_{n}\right)+\left[S_{q}^{1} \sigma_{2 i}\left(z_{1}, \cdots, z_{n-k}\right)\right.\right. \\
\left.\left.\quad+\sigma_{1}\left(z_{1}, \cdots, z_{n-k}\right) \sigma_{2 i}\left(z_{1}, \cdots, z_{n-k}\right)\right] \sigma_{2 j-1}\left(z_{n-k+1}, \cdots, z_{n}\right)\right\}^{2}
\end{array}
$$

But, (for example, see $[1$, p. 181])

$S_{q}^{1} \sigma_{2 i}\left(z_{1}, \cdots, z_{n-k}\right)+\sigma_{1}\left(z_{1}, \cdots, z_{n-k}\right) \sigma_{2 i}\left(z_{1}, \cdots, z_{n-k}\right)=\sigma_{2 i+1}\left(z_{1}, \cdots, z_{n-k}\right)$

so we have

$$
\begin{aligned}
r \beta^{*} M_{q} & =\left[\sum_{i+j=2 q} \sigma_{i}\left(z_{1}, \cdots, z_{n-k}\right) \sigma_{j}\left(z_{n-k+1}, \cdots, z_{n}\right)\right]^{2} \\
& =\sigma_{2 q}\left(z_{1}, \cdots, z_{n}\right)^{2} \\
& =r \beta^{*} \alpha^{*} p_{q} .
\end{aligned}
$$

Thus $r \beta^{*}\left(\alpha^{*} p_{q}-M_{q}\right)=\beta^{*} r\left(\alpha^{*} p_{q}-M_{q}\right)=0$ and since

$$
\beta^{*}: H^{4 q}\left(B_{Q(k)}: Z_{2}\right) \rightarrow H^{4 q}\left(B_{Q(n)} ; Z_{2}\right) \text { is } 1-1, r\left(\alpha^{*} p_{q}-M_{q}\right)=0 \text {. }
$$

Furthermore, if $\boldsymbol{Q}$ denotes the rational numbers and $\tau: H^{4 q}\left(B_{G(x)} ; \boldsymbol{Z}\right)$ $\rightarrow H^{4 q}\left(B_{G(k)} ; Q\right)$ is induced by the inclusion $Z \subset Q$, we see that

$$
\tau M_{q}= \begin{cases}\tau \tilde{p}_{q}, & q \leqq n-k / 2 \\ 0, & q>n-k / 2,\end{cases}
$$

and, as is well known, 


$$
\tau \alpha^{*} p_{q}=\left\{\begin{array}{cc}
\tau \tilde{p}_{q}, & q \leqq n-k / 2 \\
0, & q>n-k / 2 .
\end{array}\right.
$$

Thus $\tau\left(\alpha^{*} p_{q}-M_{q}\right)=0, r\left(\alpha^{*} p_{q}-M_{q}\right)=0$, and, since the only torsion in $H^{*}\left(B_{G(k)} ; Z\right)$ is of order $2, \alpha^{*} p_{q}=M_{q}$ (see $[5$, p. 112]).

We now prove that (1.3) holds. Let $\gamma: B_{S O(n)} \rightarrow B_{O(n)}$ denote the map induced by the inclusion $S O(n) \subset O(n)$. Since $\zeta$ is orientable, the associated principal bundle is induced by a map $\bar{\phi}: B \rightarrow B_{\mathbf{S O}(n)}$ with $\gamma \bar{\phi}=\phi$. In fact, $\zeta$ is induced by $\bar{\phi}$ from the bundle $\bar{\alpha}: B_{S G(k)} \rightarrow B_{S O(n)}$ and, since $\zeta$ has a cross section, there is a map $\bar{f}: B \rightarrow B_{S G(k)}$ such that the following diagram is commutative.

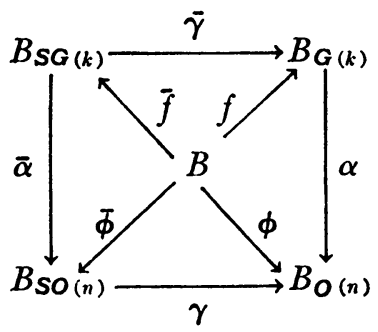

The maps $\alpha, \bar{\alpha}, \gamma, \bar{\gamma}$ are induced by inclusions.

Now, let $\bar{w}_{j}, \bar{p}_{j}, \bar{y}_{j}$ be the images of $\tilde{w}_{j}, \tilde{p}_{j}, y_{j}$ under the map $\bar{\gamma}^{*}: H^{*}\left(B_{G(k)}\right)$ $\rightarrow H^{*}\left(B_{\mathbf{S G}(\boldsymbol{k})}\right)$.

Lemma 1. If $\chi \in H^{n}\left(B_{S O(n)} ; Z\right)$ is the universal Euler-Poincaré class, then

$$
\bar{\alpha}^{*} n \chi=\delta^{*} \sum_{i+j=n-1} \tilde{w}_{i} \sigma_{j}\left(\bar{y}_{1}, \cdots, \bar{y}_{k}\right) \text {. }
$$

Proof. We prove this lemma by showing that

$$
r \bar{\alpha}^{*} n \chi=r \delta^{*} \sum_{i+j=n-1} \bar{w}_{i} \sigma_{j}\left(\bar{y}_{1}, \cdots, \bar{y}_{k}\right)
$$

and

$$
\tau \bar{\alpha}^{*} n \chi=\tau \delta^{*} \sum_{i+j=n-1} \bar{w}_{i} \sigma_{j}\left(\bar{y}_{1}, \cdots, \bar{y}_{k}\right)
$$

where $r$ and $\tau$ are as above. Thus

$$
\begin{aligned}
\bar{\beta}^{*} r \bar{\alpha}^{*} n \chi & =r \bar{\beta}^{*} \bar{\alpha}^{*} n \chi \\
& =n \sigma_{n}\left(\bar{z}_{1}, \cdots, \bar{z}_{n}\right)
\end{aligned}
$$

and

$$
\begin{aligned}
\bar{\beta}^{*} r \delta^{*} \sum_{i+j=n-1} \bar{w}_{i} \sigma_{j}\left(\bar{y}_{1}, \cdots,\right. & \left., \bar{y}_{k}\right)=S_{q}^{1} \sigma_{n-1}\left(\bar{z}_{1}, \cdots, \bar{z}_{n}\right) \\
& =\sigma_{1}\left(\bar{z}_{1}, \cdots, \bar{z}_{n}\right) \sigma_{n-1}\left(\bar{z}_{1}, \cdots, \bar{z}_{n}\right)+n \sigma_{n}\left(\bar{z}_{1}, \cdots, \bar{z}_{n}\right) .
\end{aligned}
$$


But $\sigma_{1}\left(z_{1}, \cdots, z_{n}\right)=0$ so, since $\beta^{*}$ is $1-1$,

$$
r \alpha^{*} n \chi=r \delta^{*} \sum_{i+j=n-1} \bar{w}_{i} \sigma_{j}\left(\bar{y}_{1}, \cdots, \bar{y}_{k}\right) .
$$

To prove that (2.3) holds, we need only show that $\tau \bar{\alpha}^{*} n \chi=0$ since $\tau \delta^{*}=0$. If $n$ is odd, $2 \chi=0$ so $\tau \chi=0$. If $n$ is even, we consider the following diagram.

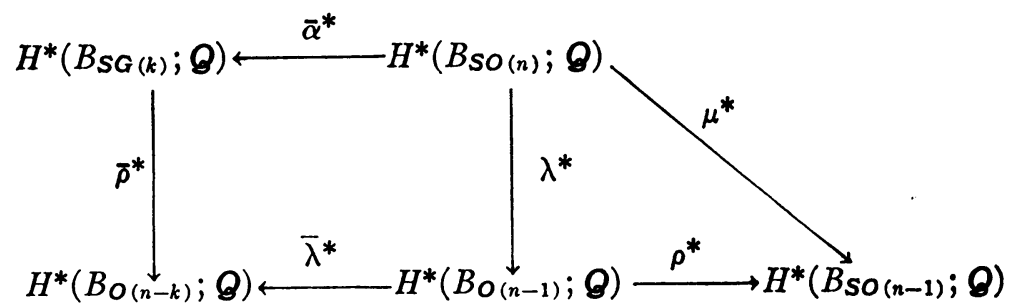

All maps are defined by the obvious inclusions. Clearly $\bar{\rho}^{*}$ is an isomorphism and, since $n-1$ is odd, $\rho^{*}$ is an isomorphism [2, p. 105]. Furthermore, it is well known that $\mu^{*} \chi=0$ so $\lambda^{*} \chi=0$. But then, $\bar{\rho}^{*} \bar{\alpha}^{*} \chi=\bar{\lambda}^{*} \lambda^{*} \chi=0$ so $\bar{\alpha}^{*} \chi=0$ and $\tau \bar{\alpha}^{*} n \chi=n \bar{\alpha}^{*} \tau \chi=0$. Thus

$$
\begin{aligned}
n \chi(\zeta) & =\bar{\phi}^{*} n \chi=\bar{f}^{*} \bar{\alpha}^{*} \chi \\
& =\bar{f}^{*} \delta^{*} \sum_{i+j=n-1} \bar{w}_{i} \sigma_{j}\left(\bar{y}_{1}, \cdots, \bar{y}_{k}\right) \\
& =\delta^{*} \sum_{i+j=n-1} u_{i} \sigma_{j}\left(x_{1}, \cdots, x_{k}\right) \\
& =\delta^{*} w_{n-1}(\zeta) .
\end{aligned}
$$

3. Orientable four-manifolds. In this section, we prove that the conditions of Corollary 1.3 are in fact necessary and sufficient for the line element parallelizability of compact orientable four dimensional manifolds. We apply this result to give an example of a four dimensional compact orientable manifold which is line element parallelizable but not parallelizable $\left({ }^{4}\right)$.

TheOREM B. Let $M$ be a compact orientable four dimensional manifold. Then, $M$ is line element parallelizable if and only if the following two conditions are satisfied.

$$
p_{1}(M)=\chi(M)=0 .
$$

(3.2) There are elements $x_{1}, \cdots, x_{4} \in H^{1}\left(M ; Z_{2}\right)$ such that $x_{1}+x_{2}+x_{3}+x_{4}=0$ and $w_{i}(M)=\sigma_{i}\left(x_{1}, \cdots, x_{4}\right), 2 \leqq i \leqq 4$.

Proof. That conditions (3.1) and (3.2) are necessary follows immediately from Corollary 2; we prove them sufficient.

Let $\phi: M \rightarrow B_{\text {So (4) }}$ be a classifying map for the principal bundle associated

(‘) Clearly, every compact orientable manifold of dimension $\leqq 3$ is line element parallelizable if and only if it is parallelizable. The Klein Bottle is an example of a nonorientable two dimensional manifold which is line element parallelizable but, of course, not parallelizable. 
with the tangent bundle of $M$. As we saw earlier, $M$ is line element parallelizable if and only if there is a map $f: M \rightarrow B_{S Q(4)}$ such that $\gamma f \simeq \phi$, where $\gamma: B_{S Q(4)}$ $\rightarrow B_{\text {SO (4) }}$ is induced by the inclusion $S Q(4) \subset S O(4)$. We now construct such a map.

Pick elements $y_{1}, \cdots, y_{4} \in H^{1}\left(B_{S Q(4)} ; Z_{2}\right)$ such that $y_{1}+\cdots+y_{4}=0$ and $\gamma^{*} w_{i}=\sigma_{i}\left(y_{1}, \cdots, y_{4}\right)$. Since $B_{S Q(4)}$ is a space of type $K\left(Z_{2}^{3}, 1\right)$, we can pick $f: M \rightarrow B_{S Q(4)}$ such that $f^{*} y_{i}=x_{i}, 1 \leqq i \leqq 4$. Then $f^{*} \gamma^{*} \chi=0=\chi(M)=\phi^{*} \chi$, $f^{*} \gamma^{*} p_{1}=0=p_{1}(M)=\phi^{*} p_{1}$ (since $H^{4}(M ; Z)$ is torsion free and $2 H^{4}\left(B_{S Q(4)} ; Z\right.$ ) $=0)$, and $f^{*} \gamma^{*} w_{2}=\sigma_{2}\left(x_{1}, \cdots, x_{4}\right)=w_{2}(M)=\phi^{*} w_{2}$ so, by [7] or [3], $\gamma f \simeq \phi$ and the theorem is proved.

We now describe the example. Let $\zeta=(M, \pi, T)$ be the nontrivial $S O(3)$ bundle over the two dimensional torus $T$ with fiber $S^{2}$. If $a, b \in H^{1}\left(T ; \boldsymbol{Z}_{2}\right)$ are generators, then $w_{2}(\zeta)=a b$ and $\chi(\zeta)=0$. To compute the characteristic classes of $M$, we need the following.

Let $\xi=(E, \pi, B)$ be an $O(n)$ bundle with fiber $S^{n-1}$ and $\tau(E), \tau(B)$ the tangent bundles to $E$ and $B$ respectively. Choosing a Riemannian metric on $E$, we can decompose $\tau(E)$ into a Whitney sum $\tau(E)=\tau_{1} \oplus \tau_{2}$ where $\tau_{1}$ is the bundle of tangent vectors along fibers in $E$ and $\tau_{2}$ the bundle of tangent vectors orthogonal to fibers. If $\xi_{1}$ is the $n$-plane bundle associated with $\xi$ and $\nu$ the trivial line bundle over $E$, one sees easily that $\left.{ }^{5}\right) \tau_{1} \oplus \nu=\pi^{-1} \xi_{1}$, the bundle over $E$ induced from $\xi$ by $\pi$. Since $\tau_{2}=\pi^{-1} \tau(B)$, it follows that

$$
\tau(E) \oplus \nu=\pi^{-1} \xi \oplus \pi^{-1} \tau(B) .
$$

Applying this to $M$, we have $\chi(M)=p_{1}(M)=0, w_{2}(M)=\pi^{*}(a b)$ $=\pi^{*}(a) \pi^{*}(b)$, and $w_{1}(M)=w_{3}(M)=w_{4}(M)=0$. Since $\chi(\zeta)=0$, we know from the Gysin sequence that $\pi^{*}: H^{*}\left(T ; Z_{2}\right) \rightarrow H^{*}\left(M ; Z_{2}\right)$ is $1-1$ so $w_{2}(M) \neq 0$ and $M$ is not parallelizable. On the other hand, if we define $x_{1}, \cdots, x_{4} \in H^{1}\left(M ; \boldsymbol{Z}_{2}\right)$ by setting $x_{1}=\pi^{*}(a), x_{2}=\pi^{*}(b), x_{3}=\pi^{*}(a+b), x_{4}=0$, we have $x_{1}+\cdots+x_{4}$ $=0$ and $w_{i}(M)=\sigma_{i}\left(x_{1}, \cdots, x_{4}\right), 2 \leqq i \leqq 4$. Thus, by Theorem $\mathrm{B}, M$ is line element parallelizable.

\section{BIBLIOGRAPHY}

1. A. Borel, La cohomologie mod 2 de certains espaces homogenes, Comment. Math. Helv. 27 (1953), 165-197.

2. - Selected topics in the theory of fiber bundles, Chicago 1954, mimeographed notes.

3. A. Dold and H. Whitney, Classification of oriented sphere bundles over a 4-complex, Ann. of Math. 69 (1959), 667-677.

4. L. Marcus, Line element fields and Lorentz structures on differentiable manifolds, Ann. of Math. 62 (1955), 411-417.

5. C. E. Miller, The topology of rotation groups, Ann. of Math. 57 (1953), 90-115.

6. H. Samelson, A theorem on differentiable manifolds, Portugal. Math. 10 (1951), 129-133.

7. W. Wu, Sur les classes caractéristiques des structures fibres spheriques, Actualités Sci. Ind. No. 1183, Hermann, Paris, 1952.

Yale UnIVERSITy, New Haven, Connecticut

(5) Apparently this fact was first observed by Wu [7, p. 86]. 